\title{
Recent Research Areas and Grand Challenges in Electronic Medical Record: A Literature Survey Approach
}

\author{
Mohammadreza Najaftorkaman \\ School of Information and Communication Technology, Griffith University \\ Gold Coast, Queensland, 4222, Australia \\ E-mail: mohammadreza.najaftorkaman@griffithuni.edu.au \\ Amir Hossein Ghapanchi \\ School of Information and Communication Technology, Griffith University \\ Gold Coast, Queensland, 4222, Australia \\ AND \\ Institute for Integrated and Intelligent Systems \\ Gold Coast, Queensland, 4222, Australia \\ E-mail: a.ghapanchi@griffith.edu.au \\ Amir Talaei-Khoei \\ Faculty of Arts and Business, University of the Sunshine Coast \\ Maroochydore DC, Queensland, 4558, Australia \\ E-mail: amirtk@usc.edu.au \\ Pradeep Ray \\ Australian School of Business (ASB), University of New South Wales \\ Sydney, New South Wales, 2052, Australia \\ E-mail: p.ray@unsw.edu.au
}

\begin{abstract}
This study undertook a literature survey to provide a taxonomy that represents research areas of Electronic Medical Record (EMR). We identified the following areas of research and classified them into eight main categories: design and implementation, evaluation, adoption, impacts, medical research, integration, EMR data design and management, and policy and standards. Even though EMR improves care quality and efficiency in a positive way, some negative perceptions by the health user community (health professionals and health service managers) should not be neglected. By categorizing EMR research articles, we reveal a clear set of grand challenges of EMR in the future education and research in health informatics, biomedical engineering, and related areas. A big picture of EMR research areas presented in this study helps health community to find scientific methods for various grand challenges in EMR.
\end{abstract}

Keywords: Electronic Medical Record, EMR, Electronic Health Record, EHR

\section{Introduction}

Healthcare processes are highly collaborative and various people from different disciplines must work together to enhance the quality of care. Achievement of this goal has been revolutionized through the use of computer-based methods. For instance, electronic medical record (EMR) is commonly used, replacing paper medical record with digital one. EMR can improve the quality of care and also reduce costs [1].

EMR has become a focus for researchers, health practitioners and policy makers because they are beneficial in enhancing operational efficiency, reducing medication errors, increasing adherence to guidelines, making the proper alerts, and decreasing costs [2]. 
The Rand research corporation [3], which is a nonprofit research organization, has estimated the potential benefits and costs of electronic health (e-health) and has described how actual health benefits can be achieved in the US healthcare system. Some of the potential benefits of e-health are efficiency savings, increased safety and better health. By adopting e-health in hospitals and doctors' offices, the annual efficiency savings can exceed $\$ 77$ billion. Moreover, if the Computerized Physician Order Entry (CPOE) system was applied in hospitals, patient safety would be increased and annual savings would be about $\$ 1$ billion. Finally, e-health can bring better health services and decrease the number of deaths by providing disease prevention and chronicdisease management systems [3].

Without doubt, EMR can have a significant influence on patient care, so it is important to categorise the various research areas involved. Although there have been numerous articles on EMR, there is still a research gap in terms of understanding the big picture of areas of EMR research.

It is important for researchers and health practitioners to be aware of these different research areas. Based on an accurate classification of EMR research, different grand challenges should be identified and resolved using scientific methods.

In response, this paper aims to classify areas of research in EMR. Therefore, researchers are generally aware of the grand challenges in research such as EMR international standards, patient-centred network models, EMR data design and management, and the various problems in EMR implementation. In addition, health practitioners are aware of Information and Communication Technology (ICT) infrastructure in EMR.

The remainder of this paper is organised as follows: Section 2 gives an overview of EMR and describes its advantages and disadvantages. Section 3 elaborates our research methodology and clarifies our criteria for including or excluding different articles. Section 4 describes the main categories of EMR research. In the section 5 , the number of papers published in different categories is presented and grand challenges faced by EMR researchers are identified. Furthermore, some recommendations for practitioners are provided with the overall aim of improving the quality and efficiency of healthcare.

\section{Research background}

Health informatics (health information systems, ehealth) is interdisciplinary and includes healthcare, information technology and computer science [4]. As a matter of fact, EMR is a primary domain of e-health [5]. In the most recent e-health research, EMR and electronic health record records (EHR) are frequently interchangeable definitions.

Medical records have been changed from traditional paper-based to digital (EMR) to ease health services in healthcare industry; nevertheless, stakeholders in healthcare are facing some challenges in applying the new health systems $[6,7]$.

Electronic health systems can be compared to their paper-based counterparts in three major aspects: from the viewpoint of patients, general practitioners (GPs) and health providers [8]. For example, in paper-based systems, patients must physically transfer their health information from one provider to another, but in an electronic system all patient information is stored in the EMR database and health providers have access to patients' data. Additionally, in paper-based system, patients with chronic diseases such as diabetes cannot update their health progress frequently, but in electronic system chronic care patients can easily update their medical records and have active role in their treatment.

Furthermore, in paper-based systems, health practitioners have some difficulties in obtaining relevant patient treatment information, but in electronic systems, patient information is stored in integrated health record database and practitioners can build better collaboration. Finally, by applying integrated electronic system, health providers access more reliable and accurate information [8].

EMR is used for financial and legal as well as clinical purposes. Data must therefore be exchanged between different people involved in the healthcare system. For this reason, the advantages and disadvantages of EMR should be carefully established because they could be a double-edged sword.

A reduction in human errors is one of the most important benefits of EMR, given that they eliminate problems of illegible writing and incorrect filing. EMR can also improve the security of medical data. Paperbased records can easily be lost, damaged or stolen, but EMR can apply information security techniques such as encryption, access control and steganography to protect medical data. Furthermore, easier access to medical data 
allows better coordination of treatment. The duplication of efforts and documents are diminished and the speed of information exchange is increased by the development of integrated medical records [9].

Even though EMR improves quality of care and patient safety in a positive way, its grand challenges should not be ignored. Based on Sittig [10] grand challenges are "fundamental scientific or technologic problems whose solutions require significant increases in our current levels of scientific knowledge and/or technical capabilities. Their solutions should significantly improve both the quality and the delivery of healthcare while decreasing its costs".

EMR is still a new concept and there are no comprehensive standards in terms of quality, efficiency and productivity. In addition, EMR is computer based, which can result in greater information security risks in terms of confidentiality, integrity and availability, so various security approaches should be applied to prevent system against computer attacks. Finally, startup cost is an important issue and healthcare providers are always faced with this challenge when allocating capital expenditure to information systems [9].

\section{Research methodology}

This study follows literature survey guidelines to achieve research objectives. Literature survey is a comprehensive review of published and unpublished research works [11]. This section elaborates research methodology of this study and identifies inclusion and exclusion of paper selection process.

\subsection{Resources Searched}

Excellence in Research for Australia (ERA) is one of the best references available to measure quality of research. It uses indicators and research experts to rank research works [12]. For this study, 10 journals in the areas of information systems, medical informatics, biomedical engineering and health informatics were selected. They are IEEE Transactions on Information Technology in Biomedicine; Computer Methods and Programs in Biomedicine; International Journal of Medical Informatics; Medical and Biological Engineering and Computing; BMC Bioinformatics; Artificial Intelligence in Medicine; BMC Health Services Research; Computers in Biology and Medicine; Journal of Biomedical Informatics; and BMC
Medical Informatics and Decision Making. These journals, ranked $\mathrm{A}^{*}, \mathrm{~A}$, or $\mathrm{B}$ in ERA, are in different databases such as ScienceDirect, Scopus and IEEE Explore.

\subsection{Search Terms}

Each online database has a specific search pattern based on its search engine framework. Titles and abstracts of papers were searched using four main keywords.

- "Electronic Medical Record"

- "EMR"

- "Electronic Health Record"

- "EHR"

\subsection{Inclusion/Exclusion Criteria}

The main purpose of this study was to categorise research on EMR into meaningful clusters. We retrieved papers published between 2011 and 2012

\subsection{Data Analysis}

First of all following search process were done. In SienceDirect online database, we used expert search and entered following search term to find suitable articles:

TITLE-ABSTR-KEY("electronic medical record") or TITLE-ABSTR-KEY(EMR) or TITLE-ABSTRKEY("Electronic health record") or TITLE-ABSTRKEY(EHR) AND LIMIT-TO(pubyr, "2012,2011") AND LIMIT-TO(cid, "272371","Journal of Biomedical Informatics")

This is an example finding articles in the Journal of Biomedical Informatics. This search term should be change based on journals' title.

Advanced search was used in Scopus database and following term was applied to find papers.

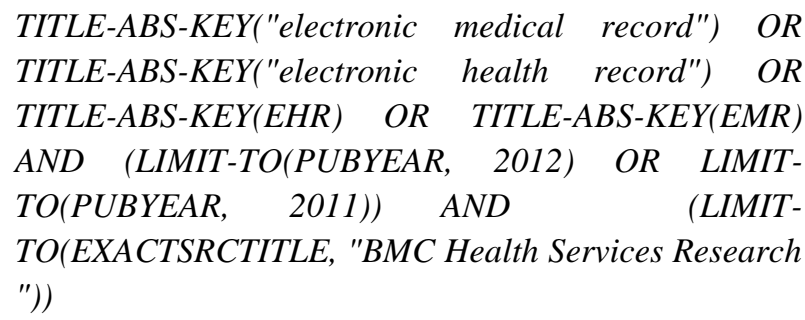

In IEEE Xplore database, the search was carried out based on the following search term and after that filter according to published date and title of journal.

(()(((("Abstract": Electronic medical record) OR Topic:EHR) OR Electronic health record) OR EMR) 
OR Topic:Electronic medical record) OR Topic:EHR) OR Topic:Electronic health record) OR Topic:EMR)

After identifying remaining papers based on inclusion and exclusion criteria and keywords, we read the titles and abstracts of the extracted articles. At this stage we tried to assign appropriate labels according to the main area of research of the papers. Unfortunately, there were some papers for which this was not clear, so we had to read the full text several times before assigning a final label. For example, some articles are closed to two or three categories.

Eventually, we grouped the papers into eight major research categories and we tried to point out the grand challenges of EMR in the final stage. Fig. 1 depicts the process undertaken to achieve our study aim.

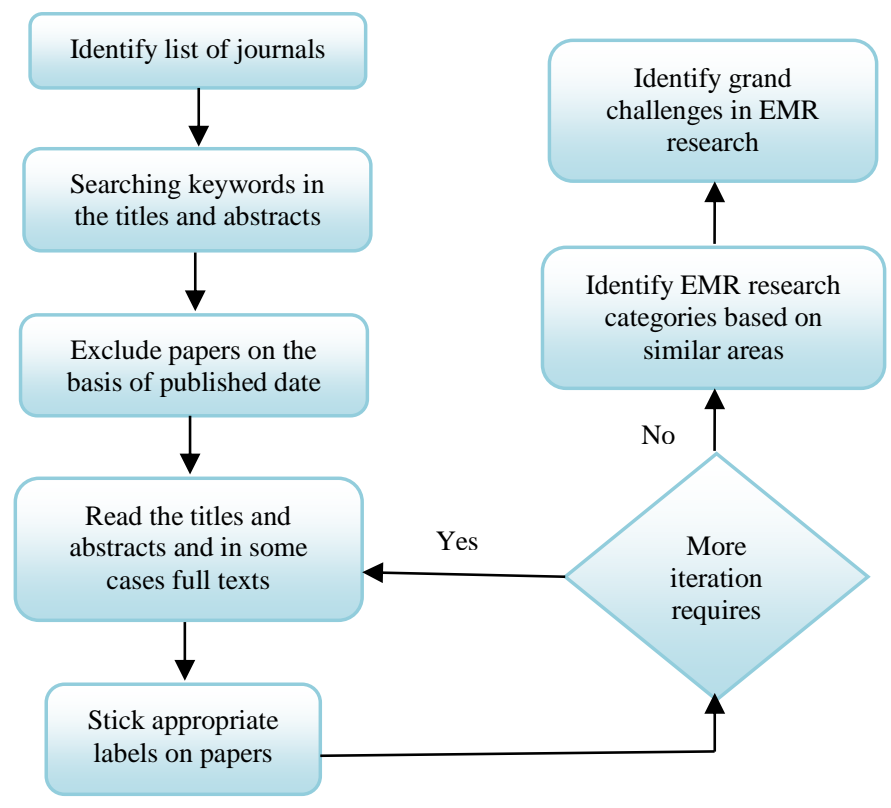

Fig. 1. Stages of the research methodology (source: developed for this study)

The Table 1 shows the number of papers (155 articles $\left.{ }^{*}\right)$ published in the 10 selected journals and the name of the database in which they found, as well as the rank of each journal according to ERA.

Table 1. List of journals included

\begin{tabular}{|l|c|c|c|}
\hline \multicolumn{1}{|c|}{ Name of Journal } & Rank & Database & $\begin{array}{c}\text { Number } \\
\text { of } \\
\text { papers }\end{array}$ \\
\hline $\begin{array}{l}\text { IEEE Transactions on } \\
\text { Information Technology in } \\
\text { Biomedicine }\end{array}$ & A* & IEEE Explore & 9 \\
\hline $\begin{array}{l}\text { Computer Methods and } \\
\text { Programs in Biomedicine }\end{array}$ & A* & Scopus & 2 \\
\hline $\begin{array}{l}\text { International Journal of } \\
\text { Medical Informatics }\end{array}$ & A & ScienceDirect & 60 \\
\hline $\begin{array}{l}\text { Medical and Biological } \\
\text { Engineering and Computing }\end{array}$ & A & Scopus & 0 \\
\hline $\begin{array}{l}\text { BMC Bioinformatics } \\
\text { Total Papers }\end{array}$ & A & Scopus & 1 \\
\hline $\begin{array}{l}\text { Artificial Intelligence in } \\
\text { Medicine }\end{array}$ & A & ScienceDirect & 0 \\
\hline $\begin{array}{l}\text { BMC Health Services } \\
\text { Research }\end{array}$ & B & Scopus & 16 \\
\hline $\begin{array}{l}\text { Computers in Biology and } \\
\text { Medicine }\end{array}$ & B & ScienceDirect & 0 \\
\hline $\begin{array}{l}\text { Journal of Biomedical } \\
\text { Informatics }\end{array}$ & B & ScienceDirect & 35 \\
\hline $\begin{array}{l}\text { BMC Medical Informatics } \\
\text { and Decision Making }\end{array}$ & B & 32 \\
\hline & & $\mathbf{1 5 5}$ \\
\hline
\end{tabular}

\section{Results}

The purpose of this study was to classify different areas of EMR research. We identified eight main categories: design and development; EMR Impacts; adoption; integration; evaluation; medical research; EMR data design and management; and policy and standards. Some of these have sub categories that are described below.

\footnotetext{
* Reference list for the literature survey can be accessed from the corresponding author's website at: https://sites.google.com/a/griffithuni.edu.au/torkman/recent-researchareas-and-grand-challenges-in-electronic-medical-record-a-literaturesurvey-approach
} 


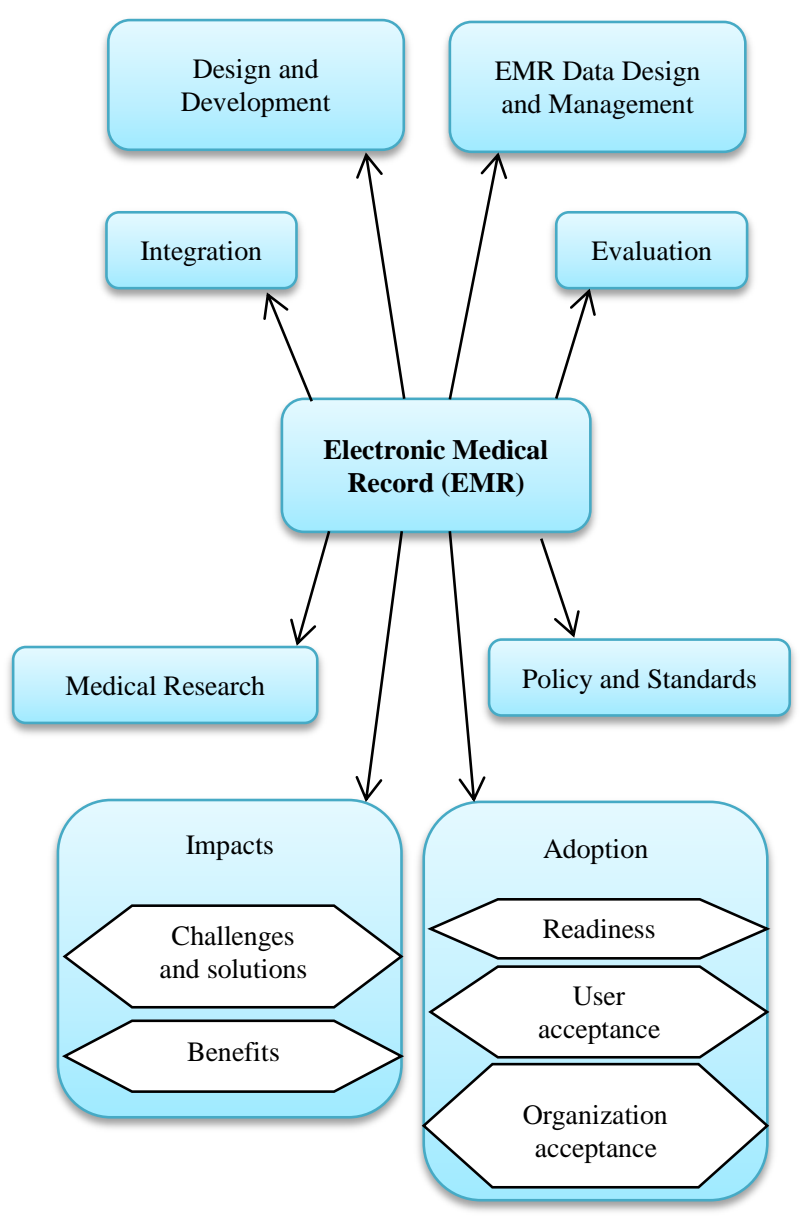

Fig. 2. Research categories of EMR (source: developed for this study)

\subsection{Design and development}

In this category, different architectures are developed to improve EMR. There were 18 papers published on developing a model, architecture, prototype, management framework, application software and medical tools.

For example, in 2011, Xiao and colleagues developed EHR architecture for methadone treatment. Their architecture has some merits, such as supporting interoperability, decision support functionality and collaboration with international health standards [13]. Moreover, in some articles, researchers have developed tools or models to improve the use of EMR [14]. For instance, the US Centers for Medicare and Medical Services have developed EHR and have tried to design a Clinical Decision Support framework, which is one of the components of their medical programme to assist with medical records.

\subsection{Evaluation}

Almost all papers in this category focus on factors affecting EMR, comparing new and old e-health systems, customised health information systems, and quality and efficiency evaluation.

For instance, McGinn assessed factors affecting Canadian record EHR based on applicability and importance [15], and evaluated these factors during implementation and maintenance. The accuracy and availability of electronic patient record systems was evaluated by Lambdin [16]. He assessed the e-health system that was implemented for the treatment of HIV in Central Mozambique. Chen and colleagues evaluated the development of EMR in developing countries, based on efficiency and efficacy evaluation and described the lack of customisation [17].

\subsection{Adoption}

There are three main subcategories in this category. The first is readiness, which focuses on organisational readiness and change management, in which different readiness factors are described to ameliorate healthcare delivery.

For example, Yoo evaluated factors that affect the successful development of electronic record systems and assessed the challenges for future systems [18]. The second subcategory is user acceptance, which tests the acceptance of systems based on end users' estimations. For example, Scholl identified challenges and strategies for the development of successful EMR in a large hospital in India for end users who lack IT skills [19]. Finally, organisation acceptance evaluates the rate of adaptation software, hardware and communication systems. For instance, Yoon tried to identify the adoption rate of EHR in Korean general hospitals [20].

\subsection{Impacts}

This category is divided to two main subcategories. The first one concentrates on the different challenges faced when implementing EMR, and offers some solutions to resolve these. The second subcategory concerns the benefits that show how EMR can improve healthcare in terms of quality, patient safety, cost, performance and computerised systems. 
For example, Walji and colleagues identified usability problems of EHR. They used multi- faceted methods to identify barriers and applied iterative design methods to solve problems [21]. Also, the potential benefits of EMR in emergency medicine were evaluated by Feufel [22].

\subsection{Medical research}

In this category, EMR database databases used for medical research. Patients' information stored in database and health providers can use it in their health experiences.

For instance, Prez-Cuevas in his paper" Evaluation of alternative standardized terminologies for medical conditions within a network of observational healthcare databases" tried to identify care indicators for type 2 diabetes using patient data extracted from EMR [23] . Moreover, Wood and colleagues have stored obesityrelated variables in an EHR database and use them to find the best obesity measurement [24].

\subsection{Integration}

All papers in this category are concerned with integration, interoperability and nationwide health information. There is an increasing demand for integrated medical record because healthcare has become more complex and fragmented into specialist areas. As a matter of fact, health practitioners' access to the accurate and relevant patients' information by using integrated EMR systems.

For instance, Leonardo and colleagues focused on integrating reasoning and clinical archetypes using Ontology Web Language (OWL) [25]. Additionally, Jose Alberto Maldonado in his paper" Using the ResearchEHR platform to facilitate the practical application of the EHR standards" presented a software platform to reach desired semantic interoperability [26].

\subsection{EMR data design and management}

EMR databases are one of the most important sources of health information system (HIS). This category describes data storage, exchange, privacy, security, data sharing, and documentation.

For example, storing details of molecular variation in DNA, RNA, and proteins and other high volume molecular data in EHR is one of the major challenges in genetic healthcare systems [27]. Furthermore, Prados-
Suarez has proposed a contextualised access system for EHR in cardiology [28].

\subsection{Policy and standards}

Unfortunately, a lack of standards is one of the common problems with health information systems. There are just 10 papers that focus on policy and standards.

For example, King assessed current policy and its development in Australia [29]. Furthermore, the challenges, strengths, limitations and uptake of the HL7 context-aware knowledge retrieval standard were described by Del Fiol's [30].

\section{Discussion}

A number of authors have discussed different aspects of EMR; however, no single article has yet given a big picture of EMR research. Health user community can use the presented big picture to know about recent research of EMR and try to find suitable solutions for EMR challenges.

In order to gain a general understanding of EMR research, we grouped all the recent studies on EMR into eight categories and presented the number of EMR studies in Fig. 3. Use of the EMR database in medical research and system evaluation had the largest number of articles (26 each). 24 papers were related to EMR data design and management, which is important in the development of EMR database. In contrast, although policy and standards have an important role to play in the implementation of EMR, only 10 papers were concerned with these aspects.

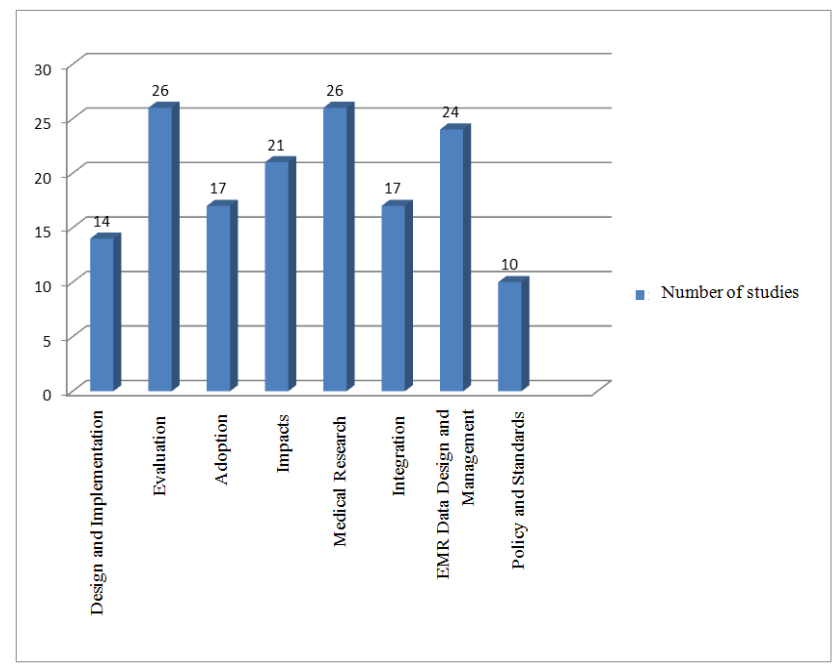

Fig. 3. Number of studies based on EMR categories (source: developed for this study) 
While, EMR has become increasingly important and tries to improve the quality and efficiency of healthcare, the challenges of EMR should not be neglected. Crossdisciplinary research and practical experiences are needed in the area of medical informatics, information system, medical engineering, public health and other related domains to find best EMR solutions.

After reviewing 155 papers related to EMR, we found that there were various grand challenges in EMR, and we identified which journals were more likely to publish papers concerned with these challenges.

\subsection{Grand challenges faced by EMR researchers}

Researchers play an important role to improve EMR systems. After investigation, we found that there are some research grand challenges faced by ERm researchers. These grand challenges are explained in the following paragraphs and include policy and standard, patient- centred network model, EMR data design and management and planning and management strategies in EMR implementation.

Firstly, we observed that there were only a few papers related to EMR policy and standards. Despite great advances in the healthcare industry, there is still a lack of integrated e-health system standards that support suitable policies and regulations to remove EMR obstacles. The development of international standards is essential; however, this is a complex task because there are various types of EMR that differ in terms of processes, cultural issues and adaptation factors. Figure 4 shows that International Journal of Medical Informatics would be beneficial for researchers who wish to know about current EMR standards and develop new one because it had the largest number of articles about this topic.

Secondly, researchers should focus on patientcentred network models of EMR because it is these that provide the foundation for data sharing between various healthcare systems. In such patient-centred systems, all patient health information is stored in integrated databases and patients do not need to transmit their physical records in a physical sense. In addition, patients can be actively involved in managing their own treatment by monitoring their EMR. The use of integrated EMR means that health service providers can achieve better collaboration and share patient treatment information more easily and reliable and accurate medical and managerial information is available. The Journal of Medical Informatics contained several papers on integrated EMR systems.

After that, more attention should be paid to EMR data design and management by researchers. Gathering, organizing, storing, presenting, and exchanging patient data are primary issues in the development of EMR. A complementary data processing model is an essential component of safe and accurate EMR. Healthcare systems contain sensitive patient data, so medical record should be stored in reliable databases that have adequate information security principles to preserve the confidentiality, integrity and availability of patent information. We found several useful papers in Journal of Medical Informatics on this topic.

Finally, there is wide agreement that EMR brings various benefits for healthcare in terms of capability, quality, and cost; nevertheless, researchers should identify the challenges related to the implementation of EMR. If they are developed and implemented without proper planning and management strategies, there is a threat to patient safety. For example, Terry has evaluated the challenges of electronic record implementation. During the first months of implementation he found that "every site had problems with medications being miscoded, items not scanning, and empty unit-dose packages being delivered to the wards." [31]. According to Fig. 4, Journal of Medical Informatics contained largest number of papers on the impacts of EMR.

EMR has come under pressures because of industrial, social, and technological changes. Existing research in EMR will not be suitable in future EMR systems. Researchers have significant roles to play in developing rigorous EMR evaluation approaches to recognize which EMR systems deliver desired benefits. By applying these kind of evaluation methods (qualitative and quantitative), evidence of effectiveness, efficiency and safety of EMR systems would be provided. 


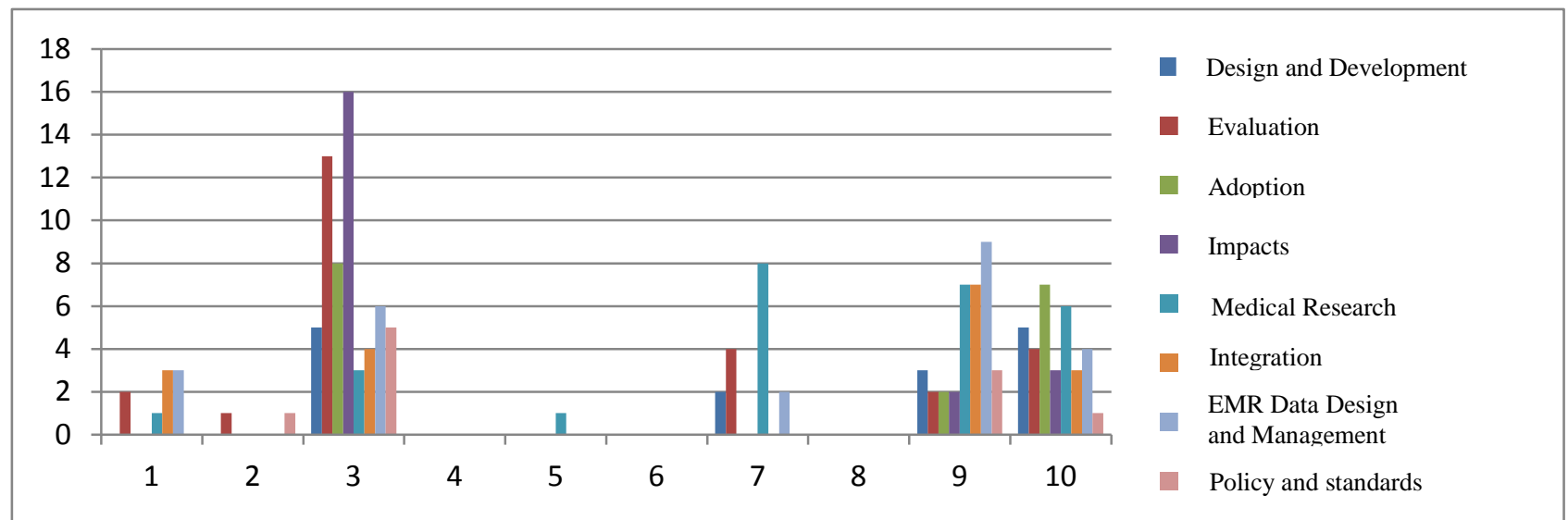

1. IEEE Transactions on Information Technology in Biomedicine

2. Computer Methods and Programs in Biomedicine

3. International Journal of Medical Informatics

4. Medical and Biological Engineering and Computing

5. BMC Bioinformatics

6. Artificial Intelligence in Medicine

7. BMC Health Services Research

8. Computers in Biology and Medicine

9. Journal of Biomedical Informatics

10. BMC Medical Informatics and Decision Making

Fig. 4. Number of studies based on EMR categories (source: developed for this study)

\subsection{Practical implications}

The number of health practitioners using of EMR in their daily practice is rapidly increasing. There are some important concepts that health practitioners should consider such as availability of ICT infrastructure, data privacy and security, staff training, and evaluation of technology acceptance in practical workplaces.

The availability of ICT infrastructure should be evaluated. The infrastructure consists of computer hardware, computer software and network structures and EMR needs these components to store and exchange medical and administrative patient information. In ICT readiness evaluation (e-readiness) of EMR, the following should be considered:

- availability of computer hardware and software in the practice

- network connectivity (The Internet or intranet)

- the bandwidth of the network connection

- IT maintenance and support

- privacy, data security and patient consent

We found valuable papers in International Journal of Medical Informatics and BMC Medical Informatics and
Decision Making that concentrate on EMR design and development.

Based on our findings, despite the wide range of EMR advantages in healthcare, its challenges should not be neglected. Medical and administrative patients' data should be accessed in the right place and at the right time and level. Unfortunately, there are knowledge management challenges in EMR implementation because of complex data processing procedures. Knowledge management concept can cover the following issues and health practitioners should be aware of these subjects during EMR implementation:

- Keeping up with new technologies

- Change management implication

- Integrated databases

- Data security

- Interoperability

- Business process and model

- Documentation management

- Execution

- Measurements

Practitioners should identify an approach for keeping up to date with new EMR developments such 
as training software and users' manuals. Productivity can be reduced at the early stage of EMR implementation because end-users need time to obtain the information required to use the new systems and adjust to the new operational framework.

Furthermore, a technology acceptance model in EMR systems should be proposed. Practitioners should contribute to understanding of EMR systems at medical centres and try to improve adoption of them. The rate of acceptance by end users reflects the proper use of EMR in healthcare. If health practitioners have problems in this area, Medical Informatics Journal contains papers relevant to system readiness, user acceptance, and organisational acceptance (Fig. 4).

\subsection{Research contributions and future research directions}

This study undertook a literature survey to provide a taxonomy that represents research areas EMR. Research areas of EMR are classified into eight main categories. This classification provides researchers and health practitioners with some knowledge of the different areas of EMR research. If they have a problem with EMR systems, they can decide to which category it belongs, according to the scheme described in this study, and locate any articles that might be of relevance to solving the problem.

It is obvious this study is not an end of something; a more important aspect is that it is the beginning of something new. Based on the finding of this study, further research must be conducted to solve various research gaps in different areas of EMR such as policy and standards, patient-centred network, EMR design and management, planning and management, availability of ICT infrastructure, and technology acceptance.

\section{Conclusion}

Despite the advantages of EMR in healthcare, there are various issues to consider during their implementation in medical practice. From survey of 155 studies, we identified different research areas of EMR. In the most recent e-health research, the terms EMR and EHR are frequently interchangeable terms; however, EHR has a broader meaning than EMR in some cases. In this study, all of these notions have same meaning and EMR is used as the most common concept. Research in EMR has been classified into eight categories: design and implementation, evaluation, adoption, impacts, medical research, integration, EMR data design and management, and policy and standards. Our findings can be used to provide an overview of EMR research that researchers and health practitioners can use in EMR design and implementation. This study indicates that before implementing the appropriate EMR systems, some particular issues should be considered as grand challenges of EMR, such as integrated EMR standards that support suitable policies and regulations, patient centred network models of EMR, complementary data processing models, proper planning and management strategies, availability of ICT infrastructure, and knowledge management models.

\section{References}

1. Bleich, H.L. and W.V. Slack, Reflections on electronic medical records: When doctors will use them and when they will not. International Journal of Medical Informatics, 2010. 79(1): p. 1-4.

2. Xue, Y., et al., Effects of electronic medical record in a Chinese hospital: A time series study. International Journal of Medical Informatics, 2012. 81(10): p. 683-689.

3. RAND Corporation, R., Health information technology: Can HIT lower costs and improve quality? Research Highlights. 2005.

4. Haux, R., Medical informatics: Past, present, future International Journal of Medical Informatics, 2010. 79(9): p. 599-610.

5. Moisil, I. and E. Jitaru, E-health progresses in Romania International Journal of Medical Informatics, 2006. 75(34 SPEC. ISS.): p. 315-321.

6. Hasman, A., Challenges for medical informatics in the 21 st century. International Journal of Medical Informatics, 1997. 44(1): p. 1-7.

7. Brender, J., C. Nøhr, and P. McNair, Research needs and priorities in health informatics. International Journal of Medical Informatics, 2000. 58-59(0): p. 257-289.

8. Deloitte organization, Queensland Health_eHealth Strategy, 12 September 2006, Retrieved February 20, 2013, from: http://www.health.qld.gov.au/ehealth/docs/eh_strat_publi c.pdf.

9. Gungor, F. Pros and Cons of Electronic Medical Records, Retrieved February 20, 2013, from: http://www.onesourcedoc.com/blog/bid/71098/Pros-andCons-of-Electronic-Medical-Records.

10. Sittig, D., Grand Challenges in Medical Informatics. . Journal of American Medical Informatics Association, 1994. 1(5): p. 412-413.

11. Literature survey guidelines by Aalto University, Retrieved February 20, 2013, from: http://www2.cs.hut.fi/ pmrg/index.cgi?id=25. 
12. Excellence in Research for Australia (ERA) research evaluation, Retrieved February 20, 2013, from: http://www.arc.gov.au/era/era_2010/era_2010.htm.

13. Xiao, L., et al., Developing an electronic health record (EHR) for methadone treatment recording and decision support. BMC Medical Informatics and Decision Making, 2011. 11(1).

14. Timbie, J.W., et al., A conceptual framework and protocol for defining clinical decision support objectives applicable to medical specialties. BMC Medical Informatics and Decision Making, 2012: p. 93.

15. McGinn, C.A., et al., Users perspectives of key factors to implementing electronic health records in Canada: A Delphi study. BMC Medical Informatics and Decision Making, 2012. 12(1).

16. Lambdin, B.H., et al., An assessment of the accuracy and availability of data in electronic patient tracking systems for patients receiving HIV treatment in central Mozambique. BMC Health Services Research, 2012. 12(1).

17. Weihua, C. and M. Akay, Developing EMRs in Developing Countries. Information Technology in Biomedicine, IEEE Transactions on, 2011. 15(1): p. 6265.

18. Yoo, S., et al., A study of user requests regarding the fully electronic health record system at Seoul National University Bundang Hospital: Challenges for future electronic health record systems. International Journal of Medical Informatics.

19. Scholl, J., S. Syed-Abdul, and L.A. Ahmed, A case study of an EMR system at a large hospital in India: Challenges and strategies for successful adoption. Journal of Biomedical Informatics, 2011. 44(6): p. 958-967.

20. Yoon, D., et al., Adoption of electronic health records in Korean tertiary teaching and general hospitals. International Journal of Medical Informatics, 2012. 81(3): p. 196-203.

21. Walji, M.F., et al., Detection and characterization of usability problems in structured data entry interfaces in dentistry. International Journal of Medical Informatics.

22. Feufel, M.A., F.E. Robinson, and V.L. Shalin, The impact of medical record technologies on collaboration in emergency medicine. International Journal of Medical Informatics, 2011. 80(8): p. e85-e95.

23. Pérez-Cuevas, R., et al., Evaluating quality of care for patients with type 2 diabetes using electronic health record information in Mexico. BMC Medical Informatics and Decision Making, 2012. 12(1).

24. Wood, G.C., et al., An electronic health record-enabled obesity database. BMC Medical Informatics and Decision Making, 2012: p. 45.

25. Lezcano, L., M.-A. Sicilia, and C. Rodríguez-Solano, Integrating reasoning and clinical archetypes using OWL ontologies and SWRL rules. Journal of Biomedical Informatics, 2011. 44(2): p. 343-353.

26. Maldonado, J.A., et al., Using the ResearchEHR platform to facilitate the practical application of the EHR standards. Journal of Biomedical Informatics, 2012. 45(4): p. 746-762.

27. Masys, D.R., et al., Technical desiderata for the integration of genomic data into Electronic Health Records. Journal of Biomedical Informatics, 2012. 45(3): p. 419-422.

28. Prados-Suarez, B., et al., Contextualized Access to Electronical Health Records in Cardiology. Information Technology in Biomedicine, IEEE Transactions on, 2012. 16(3): p. 401-412.

29. King, T., L. Brankovic, and P. Gillard, Perspectives of Australian adults about protecting the privacy of their health information in statistical databases.

30. Del Fiol, G., et al., Implementations of the HL7 ContextAware Knowledge Retrieval ("Infobutton") Standard: Challenges, strengths, limitations, and uptake. Journal of Biomedical Informatics, 2012. 45(4): p. 726-735.

31. Terry, K. Sloppy EHR Implementation Could Threaten Patient Safety. Retrieved February 20, 2013, from: http://www.informationweek.com/healthcare/electronicmedical-records/sloppy-ehr-implementation-couldthreaten/232800412. 